\title{
Long-standing Persistent Atrial Fibrillation Ablation: the Role of the Inter- and Intra-atrial Bundles
}

Russell Mitchell1', Cesar Augusto Bonilla Isaza1,*

\section{ORCID IDs}

Mitchell R (1) https://orcid.org/0000-0002-0166-009X

Bonilla C (1) https://orcid.org/0000-0002-2142-3438

\begin{abstract}
Catheter ablation has become the mainstream treatment of atrial fibrillation, but still remains a challenge in those patient with persistent and long standing persistent atrial fibrillation.

In addition of isolation of the pulmonary veins, any other areas that can trigger or perpetuate atrial fibrillation need to be isolated. Current technologies may allow to effectively deliver permanently lasting lesions, and therefore improve clinical outcomes after ablation. The specialized conduction system including the Bachmann and septopulmonary bundles, are important substrate targets for the management of atrial fibrillation. The anatomical location of these fibers, and the corresponding approach for ablation are described in this case.
\end{abstract}

KEYWORDS: Persistent atrial fibrillation; Catheter ablation; Bundles. 


\section{INTRODUCTION}

Percutaneous catheter ablation has become the mainstream treatment of atrial fibrillation, particularly for patients that are refractory, or intolerant to anti-arrhythmic medications. Newer technologies, have significantly improved the outcomes in patients with paroxysmal atrial fibrillation, but not in those with persistent atrial fibrillation ${ }^{1}$.

While the mechanism of arrhythmia in patients with paroxysmal atrial fibrillation is better understood, and isolation of the pulmonary veins from the rest of the atrium is a standardized approach; there is no consensus over the methodology for ablation in patients with persistent atrial fibrillation.

In addition of isolation of the pulmonary veins, different methods are used to modify the substrate that maintain atrial fibrillation. Single center studies suggested that targeting areas of "sick" myocardium, either with extensive linear ablation or multiple focal lesions over complex fractionated electrograms, is better than isolating the pulmonary veins alone. Those findings have not been reproduced by large randomized controlled trials $\mathrm{s}^{2,3}$.

Although there is no universal agreement on the mechanism of the arrhythmia, areas of low or null voltage, and complex and dispersed electrograms are frequently described in multiple hypotheses ${ }^{4,5}$.

Isolation of the posterior wall, in addition to the pulmonary veins, has been the most common modality used for substrate modification, in combination with discrete focal ablation of complex fractionated electrograms. Other authors routinely perform linear ablations on the mitral, and cavotricuspid isthmuses. More aggressive strategies include isolation of the left atrial appendage, the superior vena cava, and the right atrial appendage ${ }^{2,6}$.

In the following case, a comprehensive approach for catheter ablation of persistent and long-standing persistent atrial fibrillation is described.

\section{CASE REPORT}

This report presents the case of an 80-year-old gentleman with persistent atrial fibrillation of one-year duration. He had associated hypertension, mild chronic obstructive pulmonary disease, hyperlipidemia, and was a remote smoker. His left ventricular ejection fraction was 55\%, with stage I diastolic dysfunction, biatrial enlargement with a left atrium diameter of $5.8 \mathrm{~cm}$, and no significant valvular abnormalities.

He was brought for catheter ablation. The procedure was performed under general anesthesia. Systemic oral anticoagulation with apixaban was continuous until the night before of the procedure. A trans-esophageal echocardiogram ruled out atrial clots. An esophageal temperature probe was inserted, and perfectly attached to a quadripolar catheter for visualization with CARTO 3 V6 3D electro-anatomic mapping system (Biosense Webster, Inc, Diamond Bar, CA).

Then an intracardiac ultrasound catheter was inserted, from the beginning of the case, it was used to guide the insertion of the other catheters, guide the transeptal puncture, and visualize and delineate all cardiac structures, as well as the esophagus.

A three-dimensional shell of the inferior vena cava, the right atrium, and the coronary sinus was created with a Carto Thermocool Smarttouch SF ablation catheter. This process was followed by the insertion of a decapolar catheter inside the coronary sinus for pacing and recording. The presenting rhythm was atrial fibrillation. While creating the shell of the right atrium, the characteristic of electrical activation was also assessed.

After a single transeptal puncture, and advancing a steerable sheath, the mean left atrial pressure was measured at $14 \mathrm{mmHg}$. A multipolar Carto Pentaray catheter was then inserted, and used to create a three-dimensional shell of the left atrium. At the same time, the conduction properties of the left atrium during atrial fibrillation were recorded and analyzed.

The rendered left atrial volume was $256 \mathrm{ml}$, the left atrium otherwise had a normal anatomy. There were multiple areas of very fractionated, and particularly continuous small electrograms through the cycle of the tachycardia. They were densely located on the posterior wall, the floor of the left atrium, and around the base of the left atrial appendage extending towards the septum (Fig. 1 and Video 1). The amplitude of the electrograms were lower, and sometimes null in some of the same previously described areas of the left atrium, Fig. 2. 


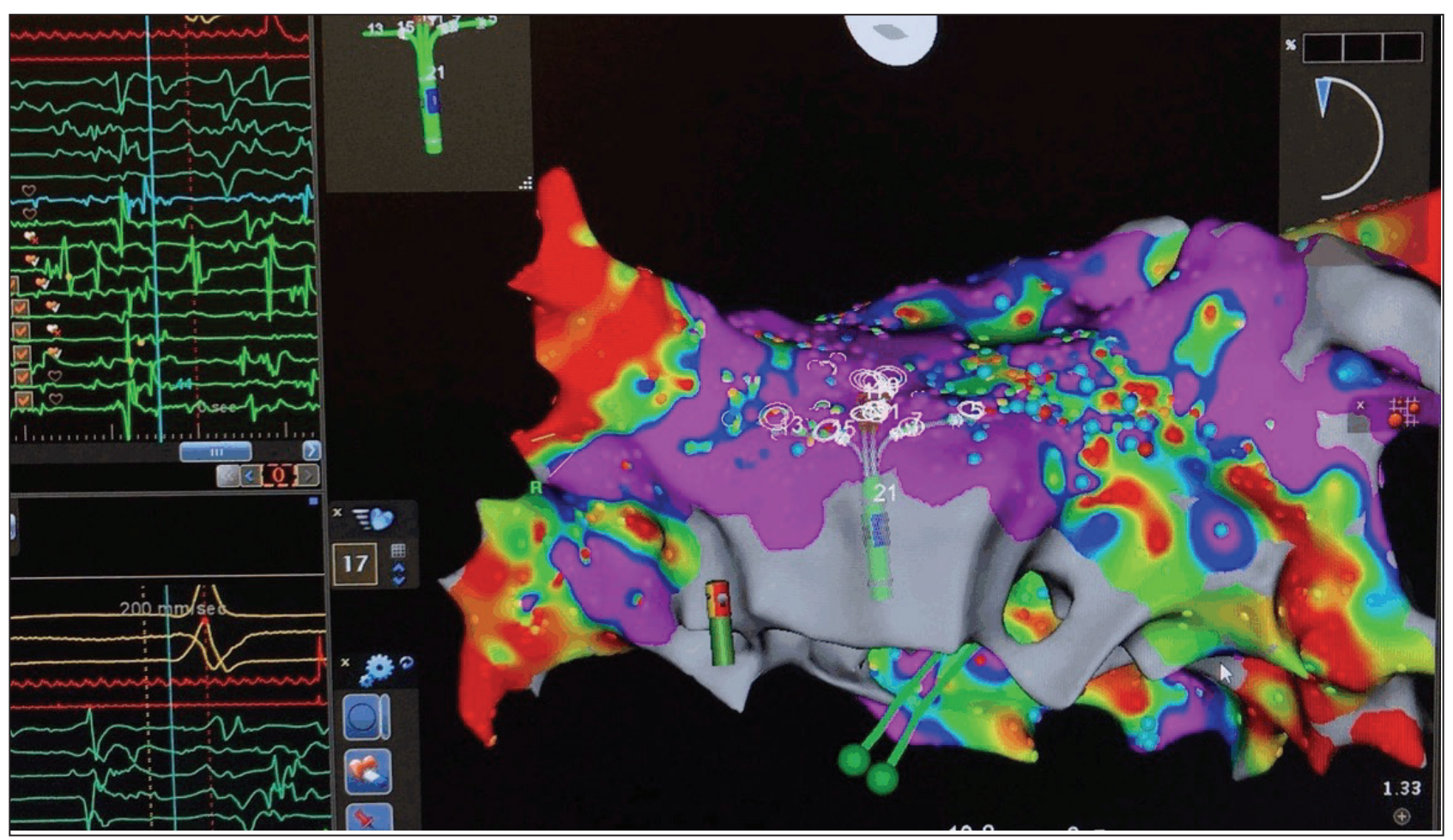

Figure 1. Fractionated electrograms. Pentaray catheter over the anterior left atrium recording multiple complex fractionated electrograms.

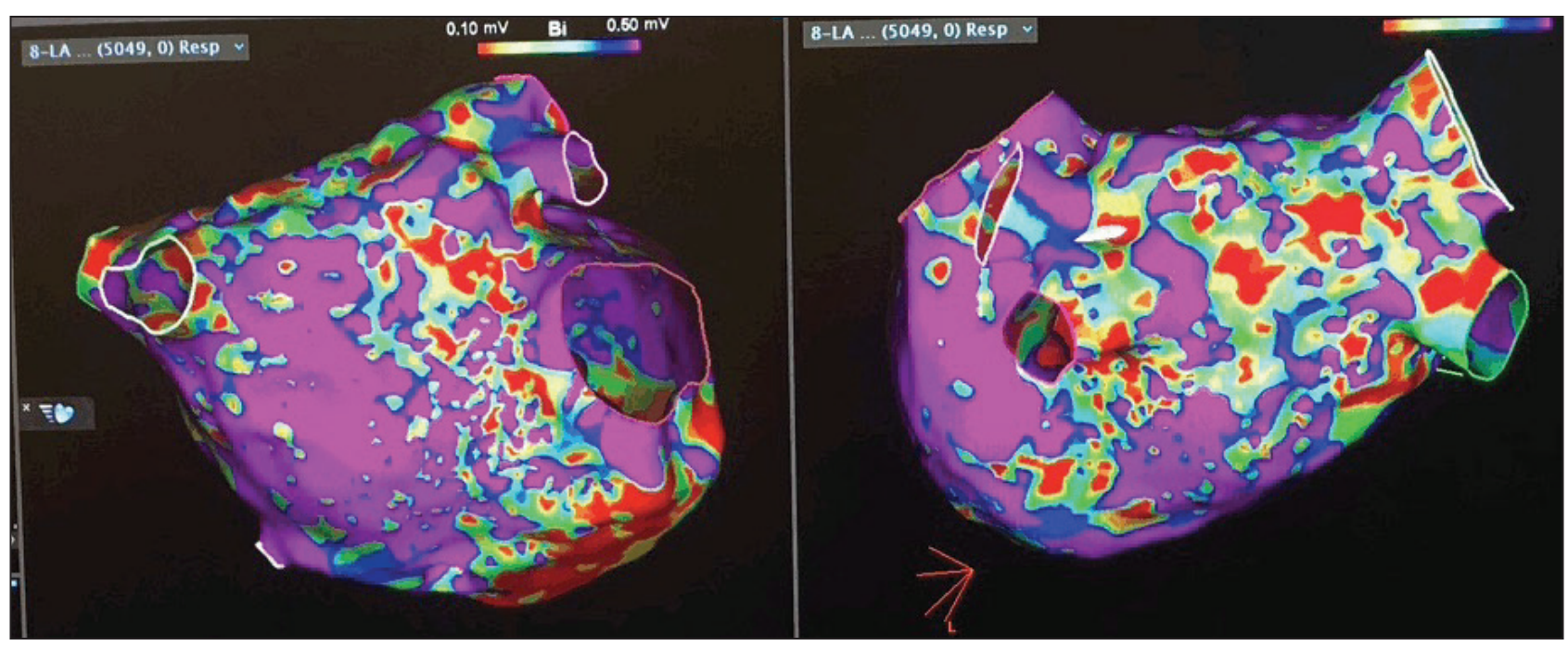

Figure 2. Left atrial voltage. Recorded left atrial voltage. The color scale for the voltage was set with voltage range from 0.1 to $0.5 \mathrm{mV}$. Voltage of less than $0.1 \mathrm{mV}$ was represented in red color. Voltage of more than $0.5 \mathrm{mV}$ was represented in magenta color.

The Pentaray catheter was exchanged for the ablation catheter through the long steerable sheath. This approach for ablation encompasses a set of goals, it combines the ablation index (AI) and impedance drop tags. A variable set of endpoints were determined depending of the anatomical location of the lesions (Fig. 3).

Equation 1 shows the AI formula with the constants replaced with letters.

$$
\mathrm{AI}=\left[\mathrm{K}^{*} \int_{0}^{\mathrm{C}} \mathrm{CF}^{\mathrm{a}}(\tau) \mathrm{P}^{\mathrm{b}}(\tau) \mathrm{d} \tau\right]^{\mathrm{c}}
$$

This formula has been integrated as an ablation lesion quality monitoring module into the automated lesion tagging software (VisiTag) in the CARTO 3 V6 3D electroanatomic mapping system (Biosense Webster, Inc, Diamond Bar, CA). 


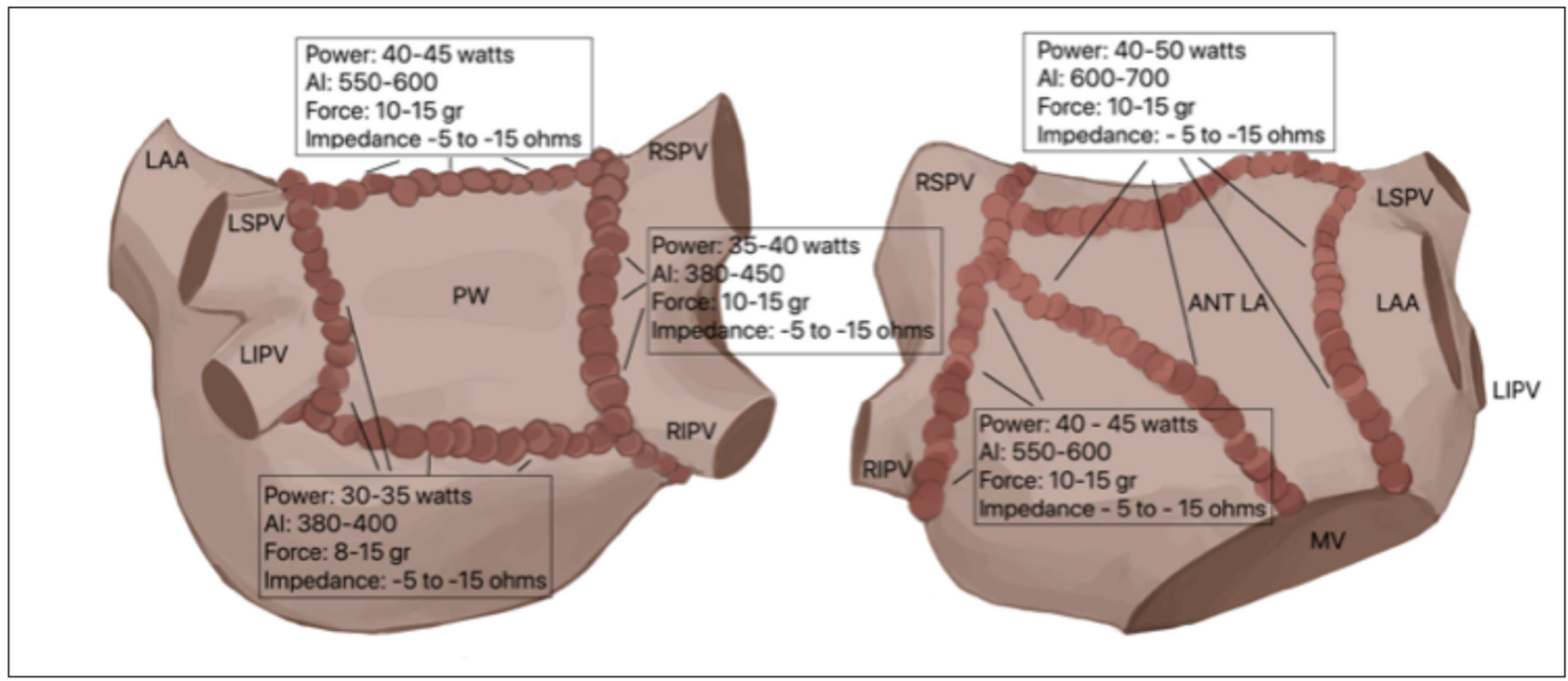

Figure 3. Ablation parameters depending of the anatomical location. PW: posterior wall; LAA: left atrial appendage; LSPV: left superior pulmonary vein; LIPV: left inferior pulmonary vein; RSPV: right superior pulmonary vein; RIPV: right inferior pulmonary vein; ANT LA: anterior left atrium; MV: mitral valve; Al: ablation index.

Electrical disconnection of the pulmonary veins from the body of the left atrium was performed. Radiofrequency lesions were applied in the power-controlled mode, using 40 to 45 watts over the ridge of the left pulmonary veins and over the septal antrum of the right pulmonary veins, and 30 to 35 watts elsewhere. During ablation of the posterior aspect of the antrum of the veins, the esophageal probe was move up and down to the level of the catheter. The lesions were immediately interrupted when the esophageal temperature started to rise. The maximum recorded esophageal temperature was $36.5^{\circ} \mathrm{C}$ during the entire case. Over the septal aspect of the right veins, phrenic nerve stimulation during high output pacing to avoid diaphragmatic paralysis was checked. The lesions were applied around the antrum of the pulmonary veins until they were completely disconnected.

Atrial fibrillation persisted, and the electrical activation in the atrium and the coronary sinus remained unchanged. A line of lesions in the roof of the left atrium were delivered, joining the two sets of lesions around the antrum of the veins at that level. The lesions were applied using 40 watts. Then, a line of lesions with a power of 30 to 35 watts were placed, over the posterior wall of the left atrium, joining the set of lesions at that level, starting at the most inferior aspect of the antrum of the left veins, and gradually withdrawing the catheter to the most inferior aspect of the antrum of the right veins. Residual potentials over the posterior wall were targeted for ablation until the posterior wall was electrically silent (Fig. 4).

Atrial fibrillation continued, and despite a slightly more organized rhythm in the coronary sinus, there was still a very disorganized, fractionated, and dispersed activation from the base of the appendage towards the intra atrial septum.

A new set of lesions were delivered with 40 to 50 watts, starting at the mitral annulus, just in front of the left atrial appendage, and extending the line of lesions to the left superior pulmonary vein where it joins the previously performed root line. Another line of ablation was delivered with the same power settings from the mitral annulus to the right superior pulmonary vein (Fig. 4).

As the lesions were delivered, atrial fibrillation in the coronary sinus started to look more organized. There were residual potentials over the anterior left atrium, they were targeted for ablation, particularly over the area of the Bachmann's bundle. Few lesions were applied over areas with residual potentials, which resulted in organization, and then termination of the tachycardia (Fig. 5 and Video 2).

An infusion of isoproterenol was started and then titrated to 15 micrograms per minute. Afterwards, isolation of all pulmonary veins was confirmed during pacing from the proximal coronary sinus, and recording in the right veins, anterior and posterior left atrium; and during pacing from the distal coronary sinus, and recording in the 
left veins, anterior and posterior left atrium. High output pacing was then performed from all pulmonary veins, anterior and posterior left atrium from multiple sites, using the Pentaray and ablation catheters to confirm exit block.

The Pentaray catheter was then placed in the left atrial appendage to record the electrograms during pacing from the proximal coronary sinus. The activation of the left atrial appendage was delayed in relationship to the distal coronary sinus as expected, but earlier than the recorded far field ventricular electrograms in the distal coronary sinus (Fig. 6).

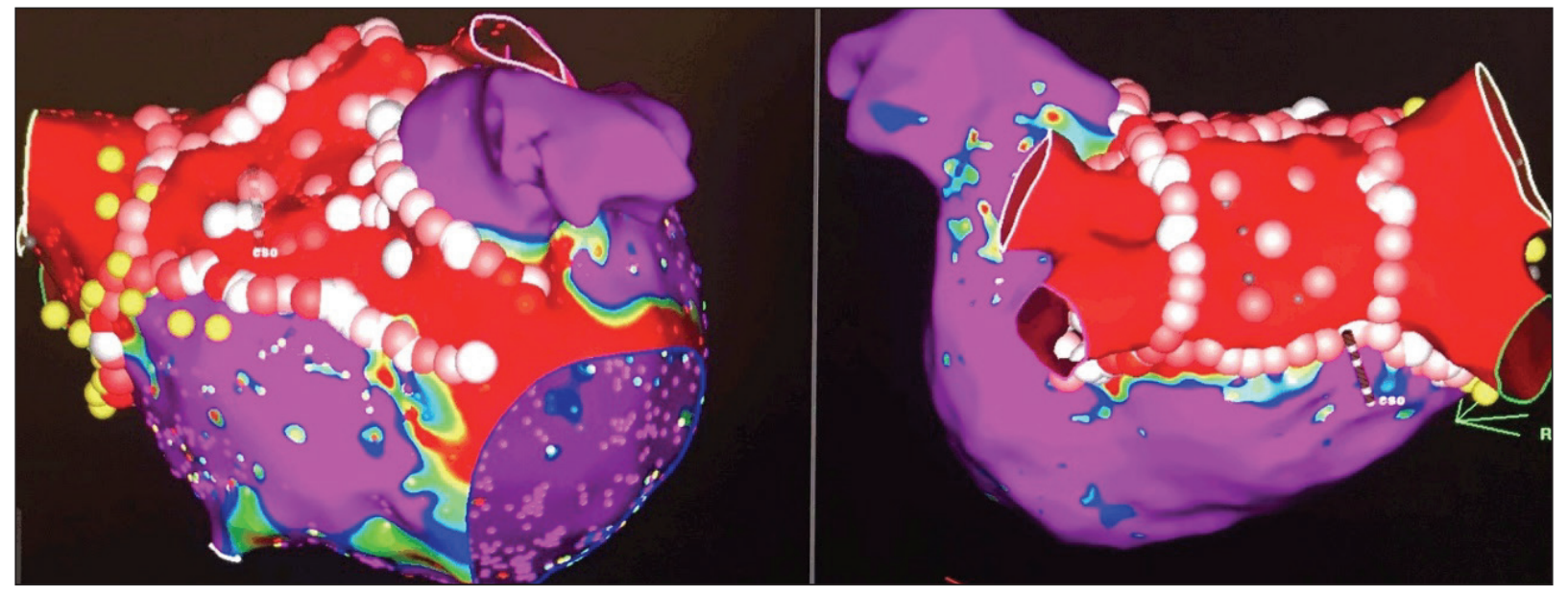

Figure 4. Post-ablation voltage and lesions set. The color scale for the voltage was set with voltage range from 0.1 to $0.50 \mathrm{mV}$. Voltage of less than $0.1 \mathrm{mV}$ was represented in red color. Voltage of more than $0.5 \mathrm{mV}$ was represented in magenta color. The lesions were tagged base on impedance drop (-5 to -15 ohms).

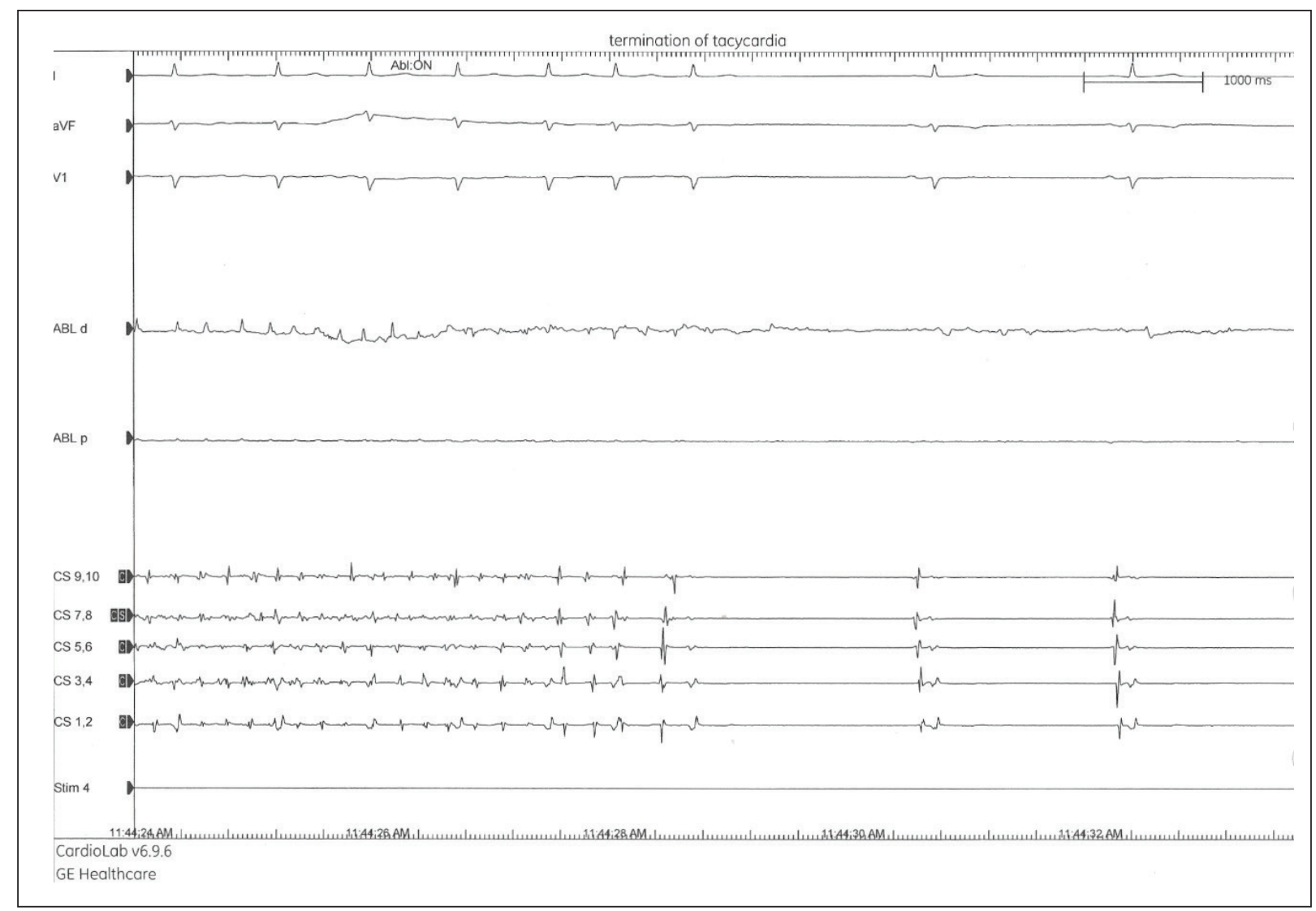

Figure 5. Intracardiac recording during ablation with the ablation and coronary sinus catheters. The arrhythmia organized and terminated during ablation over the Bachmann's bundle, which also resulted in electrical isolation of the anterior left atrium (Video 2). 


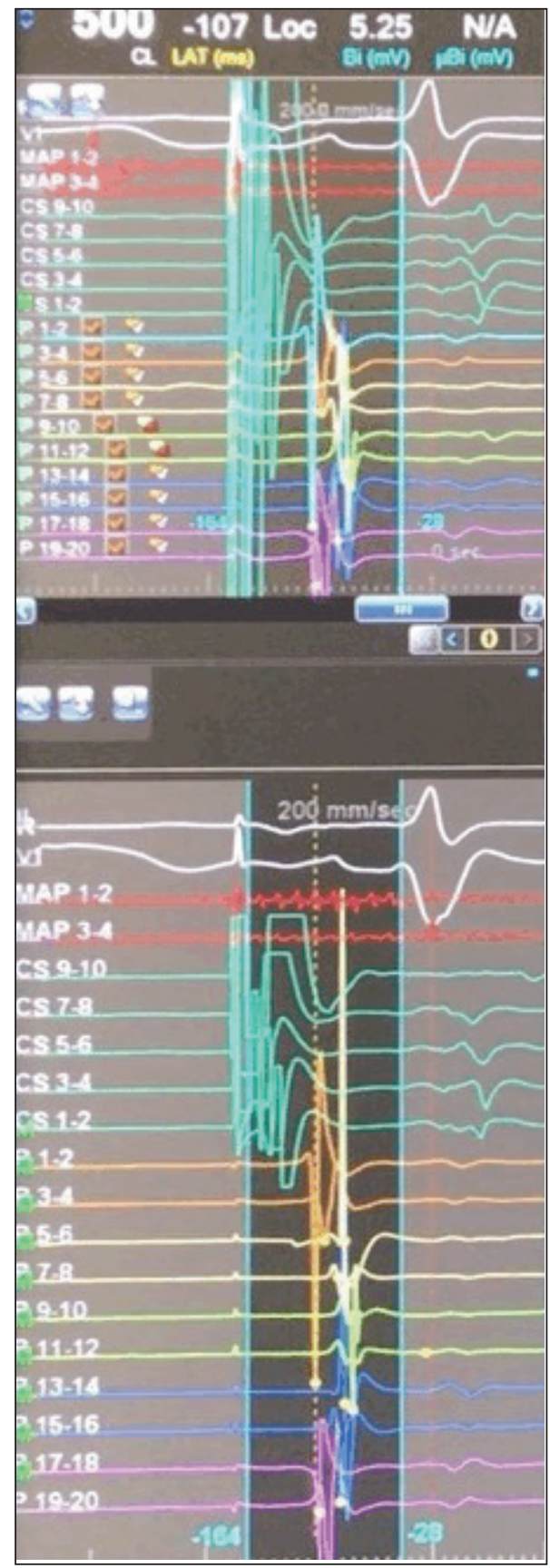

Figure 6. Intracardiac recording of the left atrial appendage with the Pentaray catheter, during atrial pacing from the proximal coronary sinus. The recorded electrograms in the left atrial appendage are delayed when compared to the distal coronary sinus, but earlier than the ventricular signals.

An electrophysiological study was performed. Atrioventricular conduction was normal. There was no evidence of dual atrioventricular nodal physiology, or accessory pathways. Aggressive atrial pacing maneuvers were delivered multiple times, including decremental atrial pacing until there was a 2:1 atrial capture $(200 \mathrm{~ms})$, and atrial programmed atrial stimulation until atrial refractoriness was achieved during a driving pacing of $400 \mathrm{~ms}$, and atrial extra-stimulus with a coupling interval of 180 ms. Any arrhythmias were induced.

The patient recovered well from general anesthesia, with no immediate complications, and discharged home the following day with systemic oral anticoagulation, but no antiarrhythmic medications. He was readmitted a couple of days later with urinary tract infection. During the hospital admission the patient did not have any arrhythmias, and was discharged in good clinical condition. He remains in normal sinus rhythm after one year. 


\section{DISCUSSION}

In many patients with persistent atrial fibrillation, most of the substrate for atrial arrhythmias involve alterations in the conduction properties over the specialized conduction system, including the Bachmann's and septopulmonary bundles and the coronary sinus. There is evidence that the Bachmann's and the septopulmonary bundles serve as bridges between the endocardium and the epicardium during atrial fibrillation 9 (Fig. 7). These abnormalities, although frequently associated with fibrosis or low voltage, can also be observed in patients with normal left atrial voltage, especially if the voltage is recorded in sinus rhythm. Conversely, the presence of low voltage in the left atrium do not necessary translate in mechanisms of arrhythmia. Special attention is focused to the patterns of propagation, and quality of the electrograms more than to the amplitude of the signals, during atrial fibrillation. Figure 8 shows an example of those signals recorded over the area of the Bachmann's bundle with the Pentaray catheter. Notice the dispersion of the recorded complex fractionated electrograms by the electrodes of the multipolar catheter over an entire cycle.

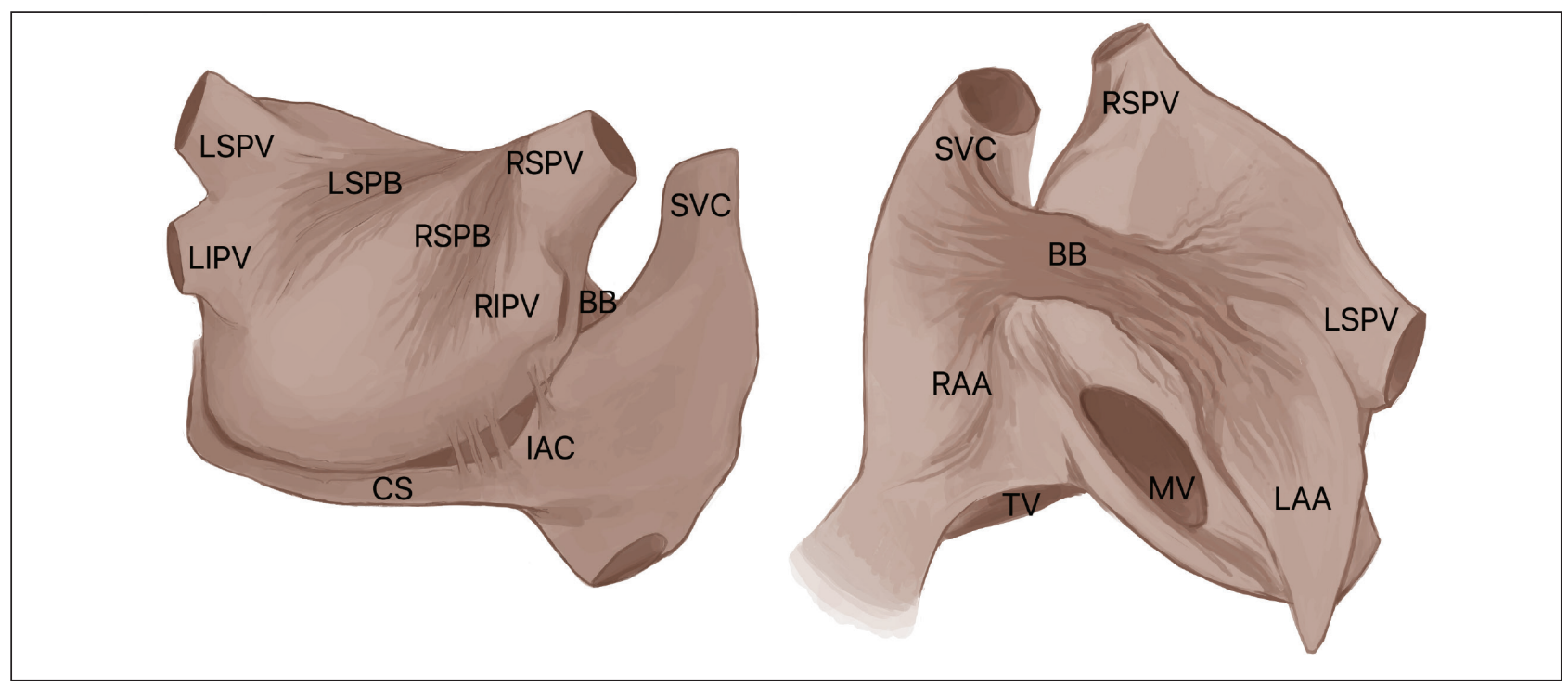

Figure 7. Atrial anatomy of the inter and intra atrial bundles.BB: Bachmann's bundle; LSPB: left septopulmonary bundle; RSPB: right septopulmonary bundle; LAA: left atrial appendage; RAA: right atrial appendage; LSPV: left superior pulmonary vein; LIPV: left inferior pulmonary vein; RSPV: right superior pulmonary vein; RIPV: right inferior pulmonary vein; SVC: superior vena cava; IVC: inferior vena cava; IAC: inter atrial connections; CS: coronary sinus; MV: mitral valve; TV: tricuspid valve.

The presence of complex, dispersed, and highly fractionated electrograms for some authors plays a very important role in the mechanism of maintenance of atrial fibrillation ${ }^{7,8}$. These types of signals are particularly densely found over the posterior wall, the base of the left atrial appendage, and the anterior left atrium towards the inter-atrial septum.

Simultaneous endocardial and epicardial mapping have demonstrated dissociated atrial activation during atrial fibrillation, mostly over the areas of the Bachmann's and septopulmonary bundles ${ }^{9,10}$. The complexity of the interconnection between the right and left atriums, and between the epicardium and endocardium during atrial fibrillation partially explains the high rate of complex, reentrant, and recurrent atrial tachy-arrhythmias that are seen in patients with persistent atrial fibrillation after they have undergone catheter ablation, especially when single lines of ablation or focal lesions over fractionated potentials have been used as strategy. It is not a surprise that the mechanism of the majority of those arrhythmias are usually found in areas corresponding to the anatomical location of the specialized bundles.

Isolation of areas with significant dispersion of electrical propagation during atrial fibrillation it seen to be more effective and results in better long-term success when properly done.

Outside the posterior and the anterior left atrium, analysis of the local electrograms over the floor of the left atrium, just above the coronary sinus, and inside the coronary sinus it is also performed, and sometimes those areas are targeted for ablation. The cavotricuspid isthmus is ablated if typical atrial flutter is documented. 


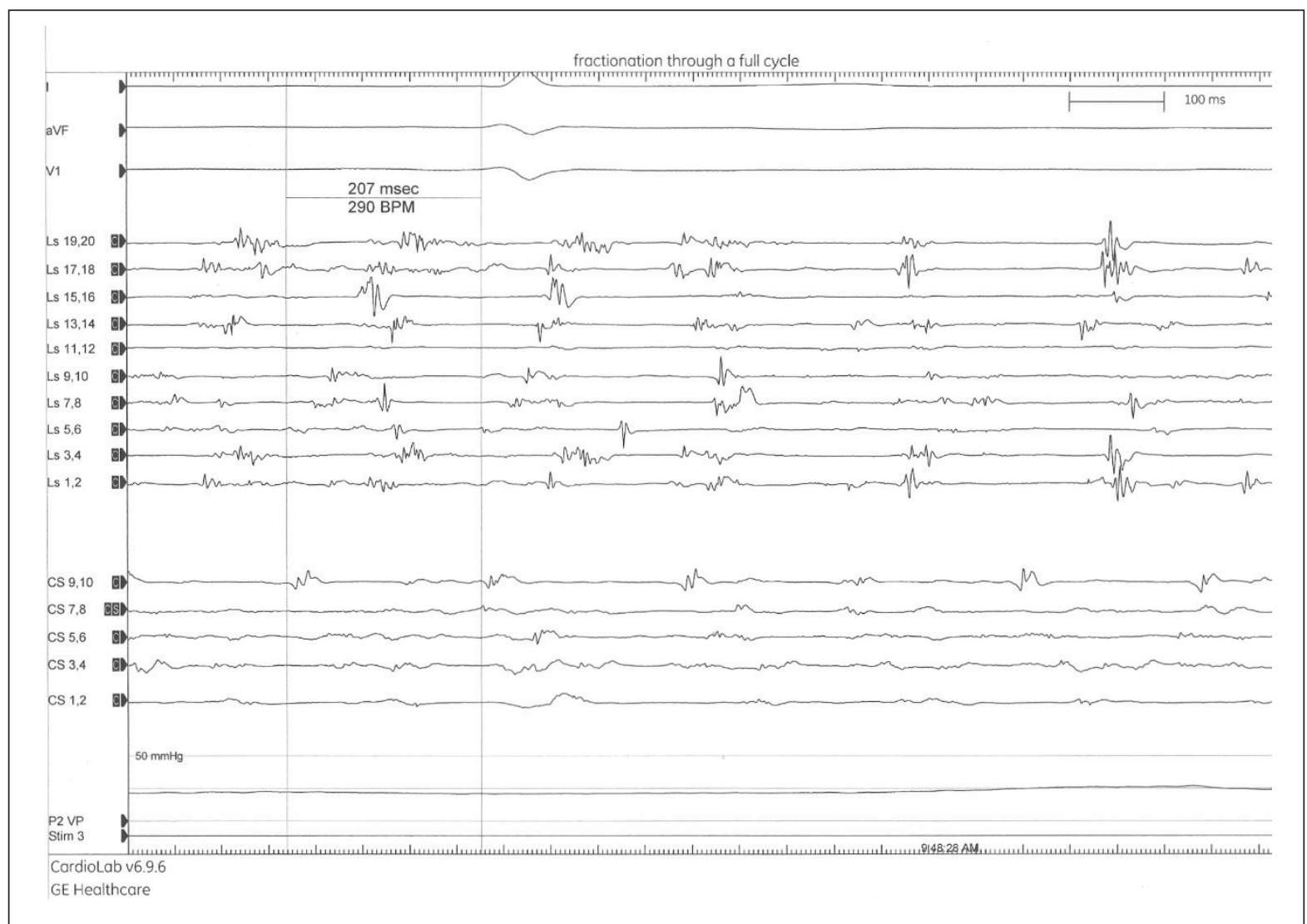

Figure 8. Fractionated electrograms: Continuous activity over the same area of interest is recorded by the Pentaray catheter. Note the dispersion of the signals over all electrodes of the catheter overlapping a full cycle in the coronary sinus.

Isolation of the left atrial appendage is linked to an increased risk of stroke and atrial thrombus, and unnecessary for most patients. Linear ablations in the lateral wall of the left atrium are rarely delivered, with the exception of the ridge of the left atrial appendage and the area of the ligament of Marshall. If isolation of the left atrial appendage is performed, a trans-esophageal echocardiogram is electively scheduled for follow-up at 6 weeks post-ablation, if the emptying velocities are significantly diminished by pulsed wave Doppler at that time, a left atrial appendage occluder is implanted.

The understanding of the variability of the atrial anatomy, tissue composition and wall thickness of the atrial tissue is fundamental in order to monitor the response to radio-frequency ablation, and to determine the power and duration of the lesions.

Organization and termination of atrial fibrillation is very common with this approach and interestingly usually occurs with a similar sequence of events and over the same anatomical locations in most of the cases. Complex arrhythmias are rarely induced after restoration of sinus rhythm, and most of the patients are discharged home without anti-arrhythmic medications.

It is important to perform an aggressive electrophysiological study under isoproterenol infusion at the end of the cases to detect residual atrial flutters. This approach, although it does not eliminate the chances of recurrent arrhythmias, probably helps to reduce it.

The most significant limitation of this technique is that the location of the Bachmann's bundle is sometimes very thick or very distant, and could be difficult to achieve complete transmural lesions. Nevertheless, the rate of recurrent atrial fibrillation is minimal, while of the residual reentrant atrial tachycardias is relatively low. The great majority of those tachy-arrhythmias are easy to map and terminate. The most common mechanism of residual tachycardias is a discrete "gap" over the anterior left atrium, especially over the area of the Bachmann's bundle, but other inter-atrial bridges are 
sometimes involved (Fig. 7). In rare occasions, some patients develop atrial arrhythmias with other complex mechanisms or epicardial connections that are difficult to eliminate without compromising the integrity of the atrioventricular conduction. In those cases, class III anti-arrhythmic drugs such as sotalol or dofetilide are very effective for long-term rhythm control.

The described technique has not been associated with an increased incidence of major complications such as atrial perforations or atrio-esophageal fistula. The procedural time is very reasonable and highly predictable, and generally well tolerated by the patients. The procedures are performed entirely without the use of fluoroscopy.

In summary, the inability to assure complete transmural lesions has been the historical limiting factor for linear ablations. New technologies, such contact force feedback, has improved the quality of the delivered lesions, but the assurance of transmural lesions remains a challenge. Multiple parameters have been used to improve the durability of the lesions without increasing the risk of complications. In addition to ablation index, observation of impedance during ablation provides useful information in terms of safety and efficacy. Impedance drop is, however, not very reliable as a surrogate of lesion formation in some patients with atrial fibrosis, but it is very useful to limit the duration of the lesions over certain anatomical areas, or when high power is used.

The development of newer and safer technologies, will hopefully allow to improve the ablation techniques, and further improve long-term success of catheter ablation in patients with persistent atrial fibrillation.

\section{ACKNOWLEDGMENTS}

The authors thank Angelica Bonilla for graphics design.

\section{REFERENCES}

1. Ganesan AN, Shipp NJ, Brooks AG, Kuklik P, Lau DH, Lim HS, et al. Long-term outcomes of catheter ablation of atrial fibrillation: a systematic review and meta-analysis. J Am Heart Assoc. 2013;2(2):e004549. https://doi.org/10.1161/JAHA.112.004549

2. Knecht S, Hocini M, Wright M, Lellouche N, O'Neill MD, Matsuo S, et al. Left atrial linear lesions are required for successful treatment of persistent atrial fibrillation. Eur Heart J. 2008;29(19):2359-66 https://doi.org/10.1093/eurheartj/ehn302

3. Verma A, Jiang C-Y, Betts TR, Chen J, Deisenhofer I, Mantovan R, et al. Approaches to catheter ablation for persistent atrial fibrillation. N Engl J Med. 2015;372(19):1812-22. https://doi.org/10.1056/NEJMoa1408288

4. Waks JW, Josephson ME. Mechanisms of atrial fibrillation - reentry, rotors and reality. Arrhythm Electrophysiol Rev. 2014;3(2):90100. https://doi.org/10.15420/aer.2014.3.2.90

5. De Groot NMS, Houben RPM, Smeets JL, Boersma E, Schotten U, Schalij MJ, et al. Electropathological substrate of longstanding persistent atrial fibrillation in patients with structural heart disease: epicardial breakthrough. Circulation. 2010;122(17):1674-82. https://doi.org/10.1161/circulationaha.109.910901

6. Di Biase L, Burkhardt JD, Mohanty P, Mohanty S, Sanchez JE, Trivedi C, et al. Left atrial appendage isolation in patients with longstanding persistent AF undergoing catheter ablation: BELIEF trial. J Am Coll Cardiol. 2016;68(18):1929-40. https://doi. org/10.1016/j.jacc.2016.07.770

7. Oral H, Chugh A, Yoshida K, Sarrazin JF, Kuhne M, Crawford T, et al. A randomized assessment of the incremental role of ablation of complex fractionated atrial electrograms after pulmonary vein isolation for long-lasting persistent atrial fibrillation. J Am Coll Cardiol. 2009;53(9):782-9. https://doi.org/10.1016/j.jacc.2008.10.054

8. Nademanee K, Oketani N. The role of complex fractionated atrial electrograms in atrial fibrillation. J Am Coll Cardiol. 2009;53(9):7901. https://doi.org/10.1016/j.jacc.2008.11.022

9. Gharaviri A, Bidar E, Potse M, Zeemering S, Verheule S, Pezzuto S, et al. Epicardial fibrosis explains increased endo-epicardial dissociation and epicardial breakthroughs in human atrial fibrillation. Front Physiol. 11:68. https://doi.org/10.3389/fphys.2020.00068

10. Groot N, van der Does L, Yaksh A, Lanters E, Teuwen C, Knops P, et al. Direct proof of endo-epicardial asynchrony of the atrial wall during atrial fibrillation in humans. Circ Arrhythm Electrophysiol. 2016;9(5):e003648. https://doi.org/10.1161/CIRCEP.115.003648 